Théologiques

Théologiques

\title{
Les dynamiques du pouvoir entre violence et justice : réflexions de théologie pratique
}

\section{R. Ruard Ganzevoort}

Volume 8, numéro 2, automne 2000

Le pouvoir

URI : https://id.erudit.org/iderudit/005007ar

DOI : https://doi.org/10.7202/005007ar

Aller au sommaire du numéro

\section{Éditeur(s)}

Faculté de théologie de l'Université de Montréal

ISSN

1188-7109 (imprimé)

1492-1413 (numérique)

Découvrir la revue

Citer cet article

Ruard Ganzevoort, R. (2000). Les dynamiques du pouvoir entre violence et justice : réflexions de théologie pratique. Théologiques, 8(2), 15-32.

https://doi.org/10.7202/005007ar
Résumé de l'article

Cet article présente un modèle psychosocial dynamique des relations depouvoir, à partir d'éléments structuraux et stratégiques. Ces élémentsstructuraux consistent en des positions et des moyens de pouvoir. Quantaux éléments stratégiques, ils sont de l'ordre de l'exercice et des motifs dupouvoir. Ce sont ces éléments qui constituent la dynamique des échanges depouvoir dans une relation entre des personnes considérées dans le contextesocial général. Le modèle permet de comprendre la relation de pouvoir dansdes situations de violence, dans un contexte d'intervention, et dans le jeu desrelations de pouvoir avec dieu. La justice et l'« empowerment " servent icid'instances critiques et normatives dans une recherche d'approchesreligieuses et théologiques plus salutaires par rapport au pouvoir et àl'intervention. 


\section{La dynamique du pouvoir entre violence et justice : réflexions de théologie pratique.}

Dr. R. Ruard GANZEVOORT

\section{Le concept de pouvoir}

Le concept de pouvoir n'est pas étranger à la théologie. De multiples façons, les théologiens et les historiens de la religion ont montré combien pouvoir et divinité sont étroitement liés, et parfois presque synonymes. La notion de "Dieu Tout-Puissant » a servi comme une sorte de critère d'orthodoxie dans de nombreuses religions et confessions chrétiennes. D'autres ont montré comment la structure de l'Église est déterminée par le pouvoir et la hiérarchie ${ }^{1}$. On peut douter que de telles notions puissent survivre au-delà du temps présent ou d'un proche avenir. Les développements historiques d'une démocratie radicalisée dans le domaine de la religion ont atteint leur apogée à notre époque (post-)moderne. Comme l'indique Don Cupitt ${ }^{2}$, le langage de tous les jours montre que notre temps en est un d'eschatologie réalisée, où Dieu est tout et en tout. Le monde est donc ce que nous en faisons et aucun pouvoir n'est accepté ou postulé au-dessus de nous. En même temps, le pouvoir demeure une force indéniable dans la conduite

1. "Whatever else their congregants believe them to be, sociologically churches are hierarchies of unequal power". SHUPE, Anson, 1998. "Introduction. The dynamics of clergy malfeasance " dans Shupe, Anson (éd.), Wolves in the fold. Religious leadership and abuses of power. New Brunswick (NJ), Rutgers University Press, p. 2 ; POLING, James N., Deliver us from evil. Resisting racial and gender oppression. Minneapolis, Fortress Press, 1996, 220 p. ; Paul BEASLEY-MurRaY, Power for God's sake. Power and abuse in the local church. Carlisle (Royaume-Uni), Paternoster, 1998, 194 p.

2. Don CUPITT, 2000. Kingdom come in everyday speech. London, SPCK, $118 \mathrm{p}$. 
humaine, peut-être de la manière la plus visible dans le comportement violent, mais également de manière visible dans la façon de faire régner la paix et la justice, au niveau des individus comme à celui des nations. Pour beaucoup de gens, un système de pouvoir perpétuel accompagné d'une légitimation est moralement et psychologiquement inacceptable. Par conséquent, nous mettrons l'accent sur la dynamique du pouvoir dans ses formes relationnelles et religieuses. Comment ce pouvoir peut-il être imaginé, analysé et interprété ? Quelles en sont les conséquences quant aux façons de faire face à la violence et à la justice ? Comment tout cela se rapporte-t-il au domaine religieux/théologique ? Ces questions vont nous guider au cours de cet article.

Le concept dynamique de pouvoir peut être compris comme la capacité à déterminer des éléments du comportement de quelqu'un d'autre, y compris la cognition et les émotions ${ }^{3}$. De façon plus abstraite, on peut le définir dans une perspective d'échange social comme étant " the level of potential cost that an actor can impose on another ${ }^{4}$. Les théories de l'échange social prennent comme point de départ l'observation suivante, à savoir qu'une bonne part de ce dont nous avons besoin et que nous considérons comme désirable dans la vie ne peut être obtenu que des autres. Les éléments de base d'une de ces théories sont les acteurs qui se comportent selon leurs intentions, les ressources disponibles, les structures d'échange et les transactions elles-mêmes. Pour notre propos, la valeur d'une perspective d'échange social réside dans l'accent qu'elle met sur le pouvoir et sur les interactions réelles entre les acteurs humains, plutôt que sur des positions et structures stables. Dans cette perspective, on propose des distinctions importantes afin de réaliser une analyse de la dynamique du pouvoir. Au cœur des théories de l'échange social se trouve la notion suivante, à savoir que la dépendance mutuelle des acteurs est la condition structurale à la fois de l'échange social et du pouvoir. Même dans les cas où l'on pourrait soutenir que le pouvoir précède la dépendance autant

3. R. Ruard GANZEVOORT, et Alexander L. VEERMAN, 2000. Geschonden lichaam. Pastorale gids voor gemeenten die geconfronteerd worden met seksueel geweld. [Corps violenté. Guide pastoral pour les communautés faisant face à la violence sexuelle.] Zoetermeer (Pays-Bas), Boekencentrum, p.40. 4. Molm, Linda D., Coercive power in social exchange. Cambridge (Royaume-Uni), University Press, 1997, p. 282. 
qu'il la suit ${ }^{5}$, le pouvoir d'une personne sur une autre est égal à la dépendance de la deuxième par rapport à la première. En ce qui concerne le pouvoir, on peut distinguer le pouvoir de récompense et le pouvoir de punition (ou coercitif), les positions structurales de pouvoir et l'usage stratégique du pouvoir, le pouvoir moyen au sein d'une relation et le déséquilibre du pouvoir entre les parties.

La notion que le pouvoir se fonde sur la dépendance et égale la dépendance a plusieurs conséquences ${ }^{6}$. Premièrement, le pouvoir est lié à une relation spécifique, non à des personnes. Il n'y a pas de personnes puissantes ou impuissantes de façon abstraite, mais seulement des relations dans lesquelles une personne a du pouvoir sur une autre. Deuxièmement, le pouvoir est un potentiel, lié à la position d'un acteur dans une structure de relations de dépendance. Le fait de savoir si ce pouvoir est exercé ou non est une autre question. Troisièmement, la quantité totale de pouvoir au sein d'une relation n'est pas fixe. L'augmentation du pouvoir d'un acteur n'implique pas la diminution du pouvoir de l'autre. Cette intuition est cruciale pour comprendre le concept d'appropriation de pouvoir («empowerment »). Quatrièmement, deux personnes peuvent avoir des relations multiples avec des équilibres de pouvoir variés. Bien qu'on puisse calculer un déséquilibre général du pouvoir entre deux personnes, les déséquilibres réels du pouvoir entre celles-ci peuvent être beaucoup plus importants pour le processus des échanges.

Aux fins de notre analyse, nous nous tournerons vers un modèle inspiré par ces théories. Ce modèle intègre les dimensions structurale et stratégique du pouvoir en distinguant quatre éléments : la position de pouvoir, les moyens de pouvoir, l'exercice du pouvoir et les motifs du pouvoir. Leurs interrelations sont présentées dans la figure $1^{7}$.

5. "Through control over vital resources [...] a group (such as the state, or a person) creates dependency on itself ». Frank R. VIVELO, Power and its consequences. A rational perspective. Lanham (MD), University Press of America, 1998, p. 39.

6. Les trois premières sont esquissées dans Linda D. Molm, Coercive power in social exchange. Cambridge (Royaume-Uni), University Press, 1997, p. 30.

7. Ce modèle a été conçu par le présent auteur, inspiré par KOOPMAN-A.M. IWEMA, Macht, motivatie, medezeggenschap. [Pouvoir, motivation, gestion conjointe.] Assen (Pays-Bas), Van Gorcum and comp., 1980 et autres, 569 p. 
FIGURE 1

Éléments structuraux et stratégiques dans la dynamique du pouvoir

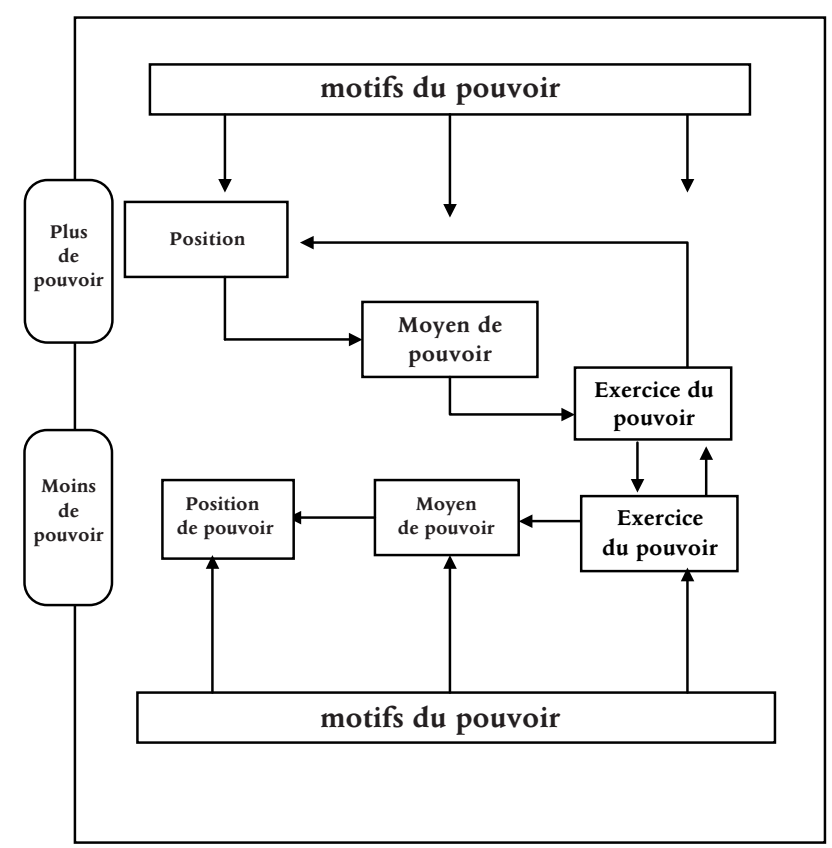

Nous décrirons ces éléments et leur apparition dans des situations de violence et de justice.

\subsection{La position de pouvoir}

La position de pouvoir renvoie à la dimension structurale. Chaque personne occupe une certaine position au sein des réseaux sociaux. Cette position est en partie déterminée par une série de facteurs démographiques tels la race, le sexe, la classe, l'âge, l'éducation, la profession et le revenu. En fonction de certaines normes (sous-)culturelles spécifiques, les déterminants qu'une personne possède en regard de chacun de ces facteurs diminuent ou augmentent sa position de pouvoir. Par exemple, dans de nombreux contextes culturels, le professeur d'université de sexe masculin et de race blanche jouit d'un grand avantage en termes de position de pouvoir par comparaison avec la femme de ménage noire.

En analysant les relations de pouvoir entre deux (ou plusieurs) personnes, nous devons prendre en considération l'éventail de relations 
plus large existant au sein des réseaux sociaux qui légitiment ou critiquent ces relations de pouvoir. Vivelo (1998, p.41) décrit l'autorité comme étant "the legitimation of power: the socially recognized "right" to exert control ». La position de pouvoir a donc un rapport avec le statut social. Au cours de notre siècle, nous pouvons discerner un changement marqué dans la nature du statut social ${ }^{8}$. Autrefois, le statut social était essentiellement attribué, accordé à une personne parce que celle-ci était née dans une position spécifique ou y avait été nommée. Ainsi, le pasteur, l'enseignant ou l'enseignante, le policier voyaient leur pouvoir légitimé, sans égard à leur performance ou à leurs mérites personnels. Aujourd'hui, le statut social est largement une conquête, basée sur ce que l'on a accompli. On doit obtenir le respect et la reconnaissance des autres, et gagner le droit d'exercer l'autorité. Ce changement a contribué à créer des relations de pouvoir plus dynamiques, comme nous avons essayé de l'inclure dans notre modèle. La position de pouvoir d'une personne par rapport à une autre n'est pas stable. Elle peut devenir plus forte ou plus faible, en fonction de facteurs extérieurs à cette relation de pouvoir et des résultats des échanges antérieurs. Cela peut influencer la quantité totale de pouvoir existant dans la relation ainsi que le déséquilibre entre les acteurs.

Dans les situations de violence, l'auteur de l'acte violent est, par définition, le plus puissant. Les personnes moins puissantes ou de puissance égale sont dépourvues de la capacité de déterminer des éléments du comportement de quelqu'un d'autre. Cependant, cet avantage en matière de pouvoir peut être partiel. On peut occuper une position de grand pouvoir et devenir une victime. La prise en otage de diplomates en est un exemple. Même dans ce cas, l'auteur de l'acte violent doit identifier ou créer une situation où il (ou elle) peut exercer un contrôle, et ce n'est que dans cette situation que le déséquilibre du pouvoir peut durer. Cet exemple montre que la description économique et politologique de l'autorité comme droit social reconnu, proposée par Vivelo, doit, jusqu'à un certain point, être adaptée lorsqu'elle est appliquée à des situations de violence interpersonnelle. La violence contredit la

8. Ce changement a été décrit en termes d'« attribué » par opposition à "réalisé ", ou d' "accordé " par opposition à "atteint ". Voir H.M. JoHnSON, Sociology. A systematic introduction. New York, Harcourt, 1960 ; SARBIN T.R. et K.E. SCHEIBE, 1983. " A model of social identity » dans Sarbin, T.R. et K.E. Scheibe (éd.), Studies in social identity, New York, 1983, 400 p. 
reconnaissance sociale. C'est précisément à cause du manque de reconnaissance sociale qu'un acte est appelé "violence ». Cependant, l'abus de pouvoir dans la violence est profondément ancré dans nos systèmes sociaux. Poling (1996, p. 8), à la suite d'autres auteurs, affirme : "The matrix of domination is a system of attitudes, behaviors and assumptions that objectifies human persons on the basis of socially constructed categories such as race, gender, class, etc., and that has the power to deny autonomy, access to resources and self-determination to those persons, while maintaining the values of the dominant society as the norm by which all else will be measured ».

Bien souvent, en particulier dans la violence domestique et sexuelle, les positions de pouvoir sont effectivement justifiées par la société; ce qui rend difficile pour les victimes d'être reconnues comme victimes de violence. Pour l'observateur, désigner la personne la plus puissante comme "auteur d'un acte violent» et ses actes comme «violence» signifierait répudier la reconnaissance auparavant accordée à cette personne et, par conséquent, reconnaître sa propre complicité dans la création de l'ordre social qui a facilité ces actes ${ }^{9}$. Étant donné le fait que la plupart des auteurs d'actes violents sont connus de la victime et ont sa confiance, nous pouvons présumer que tendances attribuées et tendances réalisées contribuent toutes deux à perpétuer les positions de pouvoir.

\subsection{Les moyens de pouvoir}

Dans les situations de violence sexuelle, on rencontre souvent une combinaison de moyens de pouvoir. Ce n'est que dans une minorité de cas que nous trouvons un seul moyen à la base d'un avantage en matière de pouvoir. Le cas classique et effrayant du viol imprévisible dans la forêt par un étranger est un exemple où seuls des moyens de pouvoir physiques sont utilisés. Cependant, il est probable que plus de $80 \%$ des cas de violence sexuelle se produisent dans le contexte de relations existantes, où l'auteur de l'acte violent dispose d'une variété de moyens de pouvoir. Les cas de violence sexuelle commise par le clergé en sont un bon exemple. Les pasteurs et les prêtres n'ont peut-être pas la même position de pouvoir qu'autrefois, mais ils bénéficient tout de même d'un avantage évident en matière de pouvoir par rapport aux victimes poten-

9. C.E. GUdORF, Victimization. Examining Christian complicity. Philadelphia, Trinity Press, 1992, 124 p. 
tielles. On trouve une utilisation typique des moyens de pouvoir par les membres du clergé dans leurs capacités verbales et relationnelles, notamment par comparaison avec des paroissiens et paroissiennes vulnérables ${ }^{10}$. De plus, ils " contrôlent » les ressources religieuses, soit symboliquement - par exemple, leur droit de proclamer la parole de Dieu et de donner l'absolution — soit littéralement — par exemple, leur droit d'administrer la communion. Les possibilités qu'a le clergé d'influencer le contexte social et le système ecclésial officiel dépassent de beaucoup celles de la victime potentielle.

Les moyens de pouvoir sont dérivés en partie de la position de pouvoir qu'occupe une personne et en partie de ses capacités, telle la prépondérance physique ou verbale. Ces capacités comprennent l'aptitude à récompenser et à punir, ou, de façon plus générale, le contrôle des ressources que l'autre considère comme désirables. Ces ressources peuvent être matérielles, émotionnelles, sociales et ainsi de suite. Les moyens de pouvoir sont alors le complément des dépendances de la personne ayant moins de pouvoir. Une personne devient plus puissante au sein d'une relation dans la mesure où l'autre dépend d'elle pour accéder à ces ressources. On peut distinguer deux mécanismes visant à diminuer ce déséquilibre du pouvoir : l'un est la réduction de la dépendance envers ces ressources chez la personne ayant moins de pouvoir ; l'autre est la réduction du contrôle exercé par la personne ayant plus de pouvoir.

Nous avons affirmé que les moins puissants avaient aussi des moyens de pouvoir à leur disposition. L'un des plus importants est peutêtre la possibilité de révéler le comportement de l'agresseur. Un autre est le fait que l'agresseur dépend de sa victime pour assouvir ses besoins (par exemple, l'intimité ou le contact sexuel). Nous avons noté que cette dépendance équivaut à un pouvoir du côté de la victime. Même si le pouvoir de l'agresseur dépasse de beaucoup le sien, la victime a en théorie le pouvoir de le priver de ressources qu'il considère comme désirables. Mais souvent, la victime n'est pas en position d'utiliser ces moyens de pouvoir parce qu'elle dépend de l'agresseur pour d'autres ressources.

10. Carrie DoEHRING, Taking care. Monitoring power dynamics and relational boundaries in pastoral care and counseling. Nashville, Abingdon, 1995, $192 \mathrm{p}$. 


\subsection{L'exercice du pouvoir}

L'exercice du pouvoir est l'usage réel de la position et des moyens de pouvoir dans des transactions spécifiques. Alors que la position et les moyens sont des éléments structuraux de la relation de pouvoir, l'exercice du pouvoir en est un élément stratégique. On peut rencontrer aussi bien des formes subtiles que des formes flagrantes, de l'exercice du pouvoir. La violence physique et la menace sont des exemples de formes flagrantes, alors que la manipulation et le charme sont des exemples de formes subtiles. Un agresseur va souvent utiliser les deux formes en alternance, récompensant et punissant la victime (visée) de manière apparemment aléatoire ${ }^{11}$. Ce caractère aléatoire menace la compréhension et le contrôle de la situation chez la victime (visée) et augmente sa dépendance. L'exercice du pouvoir comme élément stratégique a été analysé en termes de stratagèmes pour l'auteur de l'acte violent ${ }^{12}$. Dans les cas de violence sexuelle, ceux-ci consistent souvent en une planification à long terme, une mise en balance interne des désirs, des risques et des obstacles, la création d'une relation de dépendance, l'isolation de la victime visée par rapport à son réseau social et à ses ressources, une redéfinition de la relation et des actes sexuels possibles, etc.

Du côté de la victime (visée), l'exercice du pouvoir peut être appelé résistance. En fait, selon James Poling (1996, xiv), le mal et la résistance devraient être compris de façon dialectique. L'un ne peut être identifié que par rapport à l'autre. Poling définit le mal comme étant " the abuse of power in personal, social, and religious forms that destroys bodies and spirits. Evil is an abuse of power because the power of life comes from God, and all power should be used for good. Whenever power is used to destroy the bodies and spirits of God's creation, there is evil ». Il décrit la résistance au mal comme étant «a form of liberated and critical consciousness that enables persons or groups to stand against evil in silence, language and action ».

Le résultat d'un exercice stratégique du pouvoir est de renforcer ou d'ébranler les positions de pouvoir des personnes impliquées.

11. Judith L. HeRman, Trauma and recovery. New York, Basic Books, 1992, 290 p.

12. J.C. BORST, Gij zijt die man. Een onderzoek naar de pastorale zorg voor incestdaders. [Tu es cet homme. Enquête sur les soins pastoraux pour auteurs d'inceste.] Leiden (Pays-Bas), Groen, 1995, p. 79-82. 
L'exercice effectif du pouvoir dans une relation contribue à créer une position plus forte. En outre, le fait de montrer qu'on est capable d'employer les moyens de pouvoir à sa disposition, et qu'on est prêt à le faire, influence la relation de pouvoir. Quand des échanges de pouvoir antérieurs ont été efficaces pour une personne donnée, l'exercice réel des moyens de pouvoir devient moins nécessaire parce que l'autre sera convaincu que ce potentiel de pouvoir existe.

Dans notre modèle, les éléments stratégiques et structuraux s'influencent mutuellement. La structure de pouvoir née de la position et des moyens fournit les conditions pour l'exercice stratégique du pouvoir, tandis que celui-ci influence les positions et les moyens de pouvoir qui forment le point de départ des échanges futurs. Le stratagème de l'auteur de l'acte violent, tel que mentionné précédemment, consiste en un certain nombre de ces échanges, chacun d'eux visant à renforcer sa position et à affaiblir la position de la victime visée. Le but de l'auteur de l'acte violent est de saper le réseau social et les ressources de l'autre et d'accentuer sa dépendance. Souvent, la confiance de la victime en son propre jugement est constamment invalidée par l'agresseur. Cela veut dire que la victime devient encore plus dépendante, non seulement envers la récompense et la punition, mais même pour ce qui est de définir ce qui peut être compris comme une récompense ou une punition.

\subsection{Les motifs du pouvoir}

On peut comprendre les motifs du pouvoir comme étant les forces directrices qui sous-tendent les positions, les moyens et l'exercice du pouvoir. Sans ces motifs, ni les éléments structuraux ni les éléments stratégiques ne seront employés dans les échanges sociaux. On peut distinguer plusieurs motifs de pouvoir. Premièrement, le pouvoir peut être un moyen en vue d'une fin : l'acquisition de ressources considérées comme désirables. Dans les cas de violence sexuelle, le pouvoir peut être utilisé pour obtenir la satisfaction sexuelle. Dans d'autres cas, il peut servir à acquérir biens matériels, statut ou quelque chose d'autre. La victime (visée) peut recourir à des moyens de pouvoir et à l'exercice du pouvoir pour se protéger contre les menaces qu'elle perçoit. Les spectateurs, qu'ils jouent le rôle de protecteur ou de juge, peuvent utiliser le pouvoir pour pratiquer la justice. Évidemment, ces processus ressemblent à ceux de la vie économique, d'où les théories de l'échange social tirent leur origine. 
Deuxièmement, le pouvoir peut être un but en soi. Plus précisément : il peut n'avoir aucun autre but à l'intérieur de cette relation. Habituellement, ce genre de motif de pouvoir est déterminé par le besoin que l'on ressent de compenser quelque chose qui manque dans d'autres relations. Certaines personnes exercent du pouvoir dans une relation afin de neutraliser les expériences d'impuissance vécues dans d'autres relations. Il peut sembler, alors, que l'excédent de pouvoir exercé dans une relation rétablit une position de pouvoir subjective qui était menacée dans une autre relation. Cette vision homéostatique apparaît pertinente afin d'expliquer de manière partielle pourquoi certaines victimes deviennent des auteurs d'actes violents.

On peut cependant identifier un troisième genre de motifs de pouvoir qui - si on l'accepte comme valide - suggère une interprétation légèrement différente. L'exercice du pouvoir peut être exigé ou du moins validé par l'environnement social. On pourrait décrire les deux premiers types de motifs comme des forces psychologiques, lesquelles contribuent, plus ou moins rationnellement, aux aspirations de l'individu. Ce troisième type de motifs de pouvoir attire notre attention sur l'origine socio-écologique de ces motifs ${ }^{13}$. Nous pouvons nous demander pourquoi certains motifs spécifiques sont considérés comme acceptables dans certains contextes culturels? Le besoin de compenser des expériences d'impuissance n'est pas universel. En fait, si nous incluons le genre dans notre analyse, nous pouvons découvrir que ce motif de compensation est beaucoup plus présent chez les hommes que chez les femmes. Pour dire les choses crûment, les expériences d'impuissance peuvent être, pour les femmes, une affirmation radicale des messages culturels concernant leur genre et, pour les hommes, un renoncement tout aussi radical aux messages culturels concernant le leur. Si cela est vrai, on peut s'attendre à ce qu'un motif de pouvoir basé sur la compensation soit socialement et culturellement validé pour les hommes, mais non pour les femmes ${ }^{14}$.

13. Frank R. VIVELO, Power and its consequences. A rational perspective. Lanham (MD), University Press of America, 1998, p.70.

14. Comparer avec Ian M. HARRIS, Messages men hear. Constructing masculinities. Bristol (PA), Taylor \& Francis, 1995, 214 p. 


\section{Pouvoir, violence et justice}

Dans notre description des éléments d'échanges de pouvoir, nous avons utilisé à plusieurs reprises les situations de violence (sexuelle) pour illustrer les composantes abstraites de la théorie. Nous allons maintenant essayer de montrer comment cette théorie peut servir à identifier des situations de violence et aider à déterminer en quoi consistent des actions justes. Évidemment, cela présuppose une position normative, fondée sur le principe qu'il est mal d'utiliser le pouvoir au détriment des autres.

Si ce principe est accepté, nous pouvons demander comment la théorie esquissée plus haut identifie ce en quoi consiste l'utilisation du pouvoir au détriment des autres et comment il est possible de pratiquer la justice. Ces questions se posent avec acuité lorsque nous faisons face, par exemple, à un cas de violence (ou d'allégation de violence) sexuelle. Nous pouvons alors, premièrement, chercher à comprendre la relation particulière existant entre l'agresseur (présumé) et la victime supposée. On peut répertorier leurs positions et leurs moyens de pouvoir respectifs ainsi que les signaux indiquant l'exercice et les motifs du pouvoir. Dans certains cas, l'exercice du pouvoir sera très visible et la position de pouvoir ambiguë. Dans d'autres cas, on pourra retrouver des positions de pouvoir bien articulées en même temps qu'un exercice du pouvoir presque imperceptible.

Deuxièmement, faisant suite à cette analyse, nous nous attardons à nouveau au récit de la victime (supposée). La question est maintenant de savoir si l'échange de pouvoir a renforcé ou affaibli la position de pouvoir qui est la sienne. Dans notre théorie, nous avons relié cette position de pouvoir au contrôle de ressources considérées comme désirables ou à l'accès à ces ressources. Si l'échange de pouvoir a eu pour résultat une diminution du contrôle ou de l'accès, on peut définir cet échange comme étant «au détriment des autres». Cette seconde étape est essentielle, car en soi, pouvoir n'égale pas mal ou violence. Nous avons aussi noté que la quantité totale de pouvoir dans une relation n'est pas fixe. Une augmentation du pouvoir de l'un des acteurs n'implique pas une diminution du pouvoir de l'autre. On peut alors se demander si le pouvoir est utilisé sur les autres, avec les autres, ou pour les autres. On peut trouver la première façon de faire dans les structures hiérarchiques et violentes, la deuxième dans les réseaux communautaires et la troisième dans les processus de justice et d'appropriation de pouvoir. 
Troisièmement, nous pouvons nous demander quelles actions peuvent servir la justice. Afin d'évaluer les actions possibles ainsi que leurs conséquences, nous devrons examiner les positions, les moyens et l'exercice du pouvoir des personnes impliquées, tout en prenant en considération leurs motifs de pouvoir. Une analyse minutieuse de la situation peut mener à intervenir sur les positions de pouvoir, à offrir d'autres moyens de pouvoir, et ainsi de suite. Cependant, il est important de noter que la personne ou l'institution qui intervient dans la situation entre dans des relations de pouvoir aussi bien avec la victime qu'avec l'auteur de l'acte violent. Par conséquent, l'analyse doit inclure la position et les moyens de pouvoir de l'intervenant, les motifs de son pouvoir et la façon dont il exerce celui-ci en relation avec les deux parties.

L'effet de cette intervention peut même être contre-productif. On trouve des exemples de cela à tous les niveaux de pouvoir et de conflit. Les attaques de l'OTAN contre des cibles serbes en vue de forcer Milosevic à adopter une attitude plus démocratique ont conduit à une augmentation de ses actions de suppression. De même, des pasteurs peuvent faire face à des auteurs présumés d'actes de violence sexuelle domestique, et cela avec les motifs les plus nobles, et découvrir plus tard que leur action a eu pour résultat d'intensifier leurs menaces ou leur violence. Une façon de comprendre ce phénomène se trouve dans l'analyse des diverses relations de pouvoir qui sont à l'œuvre. L'intervenant possédant une position de pouvoir plus élevée, des moyens de pouvoir désirables et des motifs honorables, exerce un pouvoir dirigé vers un agresseur. Cela peut renforcer les motifs de pouvoir de l'agresseur tout en menaçant sa position de pouvoir. Afin de résoudre cette tension, l'agresseur peut essayer de protéger sa position par un exercice du pouvoir persistant et intensifié. Les victimes de cet agresseur sont alors punies pour avoir brisé le silence et demandé de l'aide, ou utilisées comme des objets pour manipuler l'intervenant.

La relation entre l'intervenant et la victime est encore compliquée, par d'autres facteurs. Même dans les cas d'intervention efficace, le message communiqué à la victime en est un de pouvoirs extérieurs. L'agresseur et l'intervenant agissent tous les deux à l'égard de la victime à partir d'une position de pouvoir élevée, avec des moyens de pouvoir étendus et un exercice du pouvoir efficace. Évidemment, leurs motifs et l'effet réalisé peuvent différer, mais de nombreuses interventions ont pour résultat non de restaurer mais de handicaper davantage les potentiels de pouvoir de la victime. C'est ici que la notion d'appro- 
priation ou de renforcement de pouvoir (empowerment) devient cruciale.

On peut décrire le renforcement de pouvoir comme les processus par lesquels des personnes moins puissantes sont encouragées à renforcer leur position de pouvoir, à developper des moyens de pouvoir et à accroître leur exercice efficace du pouvoir, en fonction de motifs de pouvoir liés à l'autonomie, à la solidarité et à la justice. Les stratégies d'appropriation de pouvoir visent à fournir ce qui est requis afin que des personnes moins puissantes deviennent à même d'écarter ce qui les menace. Plutôt que d'attaquer l'agresseur, les pratiques de renforcement de pouvoir cherchent à soutenir et à fortifier la victime. On peut distinguer trois niveaux de résultats produits par l'appropriation de pouvoir. Le premier est celui de la survie. Quand rien d'autre n'est faisable, ce niveau est justifié en ce qu'il évite l'annihilation des capacités de la victime ${ }^{15}$. Le deuxième niveau est celui du changement intérieur. Quand la situation extérieure ne peut pas (encore) être modifiée, la victime peut en arriver à un état de conscience modifié, libéré des messages insidieux de l'agresseur ${ }^{16}$. Le troisième niveau est celui de la révolution, où la situation extérieure et les structures de pouvoir qui l'accompagnent sont elles-mêmes changées ${ }^{17}$. Ces niveaux sont intrinsèquement reliés ${ }^{18}$. Évidemment, on doit si possible viser les niveaux supérieurs. Cependant, si on ne porte pas attention à la survie et au changement intérieur, la révolution non seulement échouera, mais elle pourra même nuire encore davantage à la victime. Les victimes de violence sexuelle qui se sentent obligées de porter des accusations et souffrent énormément au cours de la procédure judiciaire en sont un exemple.

15. James N. Poling, Deliver us from evil. Resisting racial and gender oppression. Minneapolis, Fortress Press, 1996, p.105.

16. Hill Collins, 1990, p. 91-114 ; James N. POling, , 1996, p.106-107. 17. Hill COllins, 1990, p. 113.

18. Dans une autre discussion, Liz KeLly, Sheila BURTON et Linda REGAN, "Beyond victim or survivor. Sexual violence, identity and feminist theory and practice » dans Adkins, Lisa et Vicky Merchant (éd.), Sexualizing the social. Power and the organization of sexuality. London, MacMillan, 1996, p. 77101 , rejettent la dichotomie ou l'ordre chronologique des identités de "victime " et de "survivant » et montrent comment les deux concepts ont des aspects significatifs et problématiques. 


\section{Pouvoir, religion et théologie}

La religion peut jouer un rôle important dans le réseau des relations de pouvoir. En fait, chaque aspect mentionné plus haut peut être appliqué au domaine religieux. Nous avons déjà noté le contrôle exercé sur les ressources religieuses dans les cas de violence sexuelle perpétrée par des membres du clergé. Nous pourrions aussi attirer l'attention sur la légitimation religieuse des positions de pouvoir, qui s'effectue par exemple grâce à un message d'obéissance qui crée chez les victimes et les spectateurs une loyauté les empêchant de résister.

Il apparaît utile de s'attarder à la relation de pouvoir entre Dieu et les humains ainsi qu'à l'interaction entre cette relation de pouvoir et d'autres relations. Dans la grille relationnelle des individus humains et des groupes, des " autres " réels aussi bien qu'imaginaires interagissent et sont mis en place par un tissu complexe de relations. Comme le note Ricoeur (1995, p. 262) : "The self is constituted and defined by its position as respondent to propositions of meaning issuing from the symbolic network ». Dans ce réseau relationnel et symbolique, Dieu opère et est perçu comme un participant, comparable aux autres participants ${ }^{19}$. Une différence importante est peut-être le fait que, d'un point de vue scientifique, les actions divines s'effectuent par l'intermédiaire d'actions, de textes et de rituels humains.

\subsection{Les relations de pouvoir entre Dieu et l'être bumain}

Notre but sera maintenant d'examiner la relation de pouvoir entre Dieu et l'être humain dans des situations de violence et de justice. Évidemment, nous ne pourrons fournir une analyse d'ensemble de la question. À cause des particularités que présente toute relation de pouvoir, des analyses spécifiques sont requises pour chaque individu religieux et chaque communauté religieuse. En un sens, Dieu est différent pour chaque personne et la relation de pouvoir entre une per-

19. Cette approche de la question de Dieu évite les discussions ontologiques et permet d'examiner les relations entre Dieu et les humains en se fondant sur la théologie et les sciences sociales. Voir par exemple : Hjalmar Sundén, Die Religion und die Rollen, Berlin, Tõpelmann, 1996 et Melvin Pollner, "Divines relations, social relations and well-being », Journal of Health and Social Behavior 30, p. 92-104 
sonne et Dieu dépendra de la position, des moyens, de l'exercice et des motifs du pouvoir humain, ainsi que de la perception de la position, des moyens de pouvoir, de l'exercice et des motifs du pouvoir divin. On peut toutefois formuler certaines remarques globales, fondées sur le modèle esquissé plus haut, tout en invitant les lecteurs à considérer les aspects spécifiques de leur propre relation de pouvoir avec Dieu ainsi que de celle des autres avec Dieu.

Nos remarques globales ont pour point de départ la vie religieuse des victimes de violence sexuelle. Pour un grand nombre d'entre elles, Dieu leur a été présenté par l'intermédiaire d'un cadre narratif religieux de souveraineté, de jugement et d'action autonome. On ne peut rendre sa divinité transcendante dépendante des êtres humains, et même s' $\mathrm{Il}^{20}$ répond à la prière, Il a toujours la liberté de choisir s'Il se laisse déterminer par les humains ${ }^{21}$. Dieu - tel que ces personnes en sont venues à Le connaître - est dans une position de pouvoir absolu. Cela veut dire que dans la relation de pouvoir entre Dieu et les humains, ces derniers sont dans une position de dépendance et d'impuissance absolues.

De la même façon, les moyens de pouvoir de Dieu sont illimités. Si le pouvoir est effectivement compris comme la capacité de déterminer des éléments du comportement de quelqu'un d'autre, y compris la cognition et les émotions, alors Dieu a à sa disposition des moyens de pouvoir infinis. Non seulement contrôle-t-Il toutes les ressources que l'individu religieux considère comme désirables - l'intimité, le confort, les récompenses éternelles et ainsi de suite - mais Il détermine aussi notre cœur et notre âme par l'œuvre de l'Esprit Saint qui habite en nous. Dans les théologies réformées radicalisées, cela a mené à une vision élaborée de la prédestination dans laquelle toute contribution humaine à la relation avec Dieu est niée ou réinterprétée comme étant l'œuvre de Dieu. Puisque Dieu jouit d'une position et de moyens de pouvoir illimités, l'exercice du pouvoir devient unidirectionnel. La contribution de l'individu humain est réduite à pratiquement rien, et la résistance à Dieu est passible de condamnation.

20. Dans cette ligne de pensée, Dieu est habituellement perçu comme masculin.

21. Voir une discussion sur ce point dans Kathryne E. TANNER, God and creation in Christian theology. Tyranny or empowerment. Oxford (Royaume-Uni), Basil Blackwell, 1998. p.96 et suiv. 
Aussi négatif et caricatural que peut sembler ce portrait, il s'agit probablement de la relation de pouvoir Dieu - être humain dominante dans de nombreuses confessions chrétiennes. Elle apparaît comme la principale image de Dieu à laquelle les victimes de violence et leurs défenseurs font référence dans le contexte de leur résistance. Une autre image importante se trouve dans la souffrance du Christ. Alors que le Dieu tout-puissant n'est pas un objet d'identification, mais renforce plutôt l'impuissance, le Christ souffre parce qu'Il se substitue aux humains dans la rédemption. Cependant, cela implique souvent une glorification de la souffrance et de l'état de victime ${ }^{22}$.

\subsection{Dieu, la justice et l'appropriation de pouvoir}

Les messages dominants concernant Dieu et sa relation de pouvoir avec les humains sont donc discutables quand il s'agit de leur impact sur les personnes victimisées. Ces messages peuvent même renforcer les sens et les effets de la violence. La violence aussi bien que les messages dominants sur Dieu sapent les positions et les moyens de pouvoir des victimes ainsi que leur exercice du pouvoir. Tous deux forcent la victime à se diriger vers la capitulation et la loyauté. Cela dit, nous devons souligner clairement que dans la Bible et dans la plupart des traditions chrétiennes, on trouve de fortes tendances caractérisées par une résistance à la violence et une volonté d'instaurer la justice. Pour cette raison, certains ont affirmé catégoriquement que le problème n'est pas causé par le message chrétien infaillible, mais par des gens faillibles et, en fait, pécheurs qui se servent du message de manière abusive. Même vrai, cet argument soutient la relation de pouvoir problématique Dieu - être humain décrite précédemment. En essayant de défendre le message (et Dieu), on blâme les humains.

Comme dans le cas d'une intervention humaine, il nous faut une analyse plus profonde de la façon dont les messages portant sur le pouvoir de Dieu interagissent avec les relations de pouvoir entre

22. Joanne CARLSON BROWN, et Rebecca PARKER, "For God so loved the world? » dans Adams, Carol J. et Marie M. Fortune (éd.), Violence against women and children. A Christian theological sourcebook. New York, Continuum, 1995, p. 36-5 
agresseur et victime. Cette analyse devrait comprendre le fait que les textes religieux sont vulnérables à une manipulation ayant pour effet que les victimes ne peuvent recourir aux éléments religieux potentiellement libérateurs. Le lien entre pouvoir divin et justice divine en est un bon exemple. S'attendre à ce que Dieu fasse justice peut avoir du sens pour les personnes souffrant d'injustice et de violence. Cependant, cette attente est souvent traduite en termes de péché et de pardon ou d'absolution. Dans le catholicisme et le protestantisme traditionnels, le péché et le pardon sont tous deux compris comme étant surtout à l'œuvre dans la relation entre Dieu et le pécheur. La victime de ces péchés devient presque invisible. Cela a pour effet la rédemption de l'agresseur et l'annihilation de la victime.

Parallèlement aux efforts visant à réorganiser nos systèmes juridiques en termes de justice réparatrice ${ }^{23}$, en mettant l'accent sur des actes réparateurs à l'égard de la victime plutôt que de l'État, une réinterprétation des sources bibliques et religieuses est nécessaire. Nous avons besoin d'une compréhension de la justice et du pouvoir de Dieu qui soit source de réappropriation du pouvoir (empowering). Les théologies de la libération ayant pour base le féminisme, la conscience noire et les communautés gaies et lesbiennes peuvent contribuer à cette compréhension. Il est également possible d'y trouver de nombreux récits libérateurs, allant du récit de l'Exode aux prophètes s'adressant aux puissants, en passant par les actes d'exorcisme de Jésus.

Le concept de renforcement de pouvoir par l'action de Dieu (empowerment by God) doit atténuer la juxtaposition de Dieu et des humains. Au lieu d'une théologie implicite qui enseigne que le pouvoir souverain de Dieu ne permet pas la dépendance ou le pouvoir humain, il nous faut des théologies de pouvoir fondé sur la collaboration, où Dieu exerce son pouvoir de manière à faciliter le pouvoir humain, en particulier chez les moins puissants ${ }^{24}$. La notion d'appropriation de pouvoir inclut le postulat moral que le pouvoir destructeur est mauvais.

23. Virginia MACKEY, Restorative justice. Toward nonviolence. Louisville (KY), Presbyterian Criminal Justice Program, 1997, 87 p.

24. Ce concept de pouvoir est davantage féminin. Voir HAMPSON, D., " On power and gender ", dans A. Thatcher, et E. Stuart (éd.) Christian perspectives on sexuality and gender, Grand Rapids, Eerdmans, 1996, 478 p. 
Pour pratiquer la justice, nous devons donc donner une priorité herméneutique et morale aux personnes qui sont devenues victimes ${ }^{25}$. La première tâche des théologiens à cet égard est de rendre les autres plus conscients des effets, sur les relations de pouvoir entre les humains, de leur raisonnement abstrait quant au pouvoir de Dieu. La deuxième tâche est d'aider la communauté chrétienne à construire et à reconstruire des images et des messages de Dieu qui soient source d'autonomie et de pouvoir, rendant possible pour les victimes aussi bien que pour les agresseurs de vivre une relation salutaire avec Dieu, c'est-à-dire juste, libre et véritablement humaine.

\section{RÉSUMÉ}

Cet article présente un modèle psychosocial dynamique des relations de pouvoir, à partir d'éléments structuraux et stratégiques. Ces éléments structuraux consistent en des positions et des moyens de pouvoir. Quant aux éléments stratégiques, ils sont de l'ordre de l'exercice et des motifs du pouvoir. Ce sont ces éléments qui constituent la dynamique des échanges de pouvoir dans une relation entre des personnes considérées dans le contexte social général. Le modèle permet de comprendre la relation de pouvoir dans des situations de violence, dans un contexte d'intervention, et dans le jeu des relations de pouvoir avec dieu. La justice et l' « empowerment " servent ici d'instances critiques et normatives dans une recherche d'approches religieuses et théologiques plus salutaires par rapport au pouvoir et à l'intervention.

\section{ABSTRACT}

This paper presents a dynamic psycho-social model of power relations, consisting of structural and strategic elements. Structural elements of power relations are power position and power means. Strategic elements are power execution and power motives. These elements constitute dynamically the power transaction in a relation between persons, connected to the wider social network. The model assists in understanding power relations in situations of violence, effects of intervention, and the interaction to power relations with God. Justice and empowerment serve as critical and normative notions in search of more salutary religious and theological approaches to power and intervention.

25. James N., Poling, Deliver us from evil. Resisting racial and gender oppression. Minneapolis, Fortress Press, 1996, p.103. 